\title{
Population characteristics, space use and habitat associations of the seahorse Hippocampus reidi (Teleostei: Syngnathidae)
}

\author{
Ierecê Lucena Rosa*, Tacyana P. R. Oliveira*, André L. C. Castro*, \\ Luiz E. de Souza Moraes**, Josias H. A. Xavier*, Mara C. Nottingham***, \\ Thelma L. P. Dias*, Leonardo V. Bruto-Costa****, Maria E. Araújo****, \\ Alesandra B. Birolo*****, Ana C. G. Mai* and Cassiano Monteiro-Neto**
}

This paper provides a case study of a threatened seahorse species, Hippocampus reidi, highlighting the importance of using ecological information to assist conservation and management initiatives. Underwater visual sighting data ( $50 \times 2 \mathrm{~m}$ transect) gathered along the NE, SE and S portions of the Brazilian coast revealed an unequal distribution across localities, perhaps related to harvesting pressure, and a mean density of 0.026 ind. $\mathrm{m}^{-2}$. Our findings suggest some restricted spatial use by $H$. reidi, which was consistent with its estimated home range, and with re-sighting of specimens. Reproduction was recorded year-round, however productive peaks may exist. Components of habitat structure mostly used as anchoring points were mangrove plants, macroalgae, cnidarians, seagrass, sponges, and bryozoans. Conservation recommendations include: further characterization and mapping of habitats; assessment of availability and condition of microhabitats in selected areas, and studies on dispersal routes during initial life stages.

Neste artigo apresenta-se um estudo de caso de uma espécie ameaçada de cavalo-marinho, Hippocampus reidi, enfatizando a importância de utilizar informações ecológicas nas ações de conservação e manejo. Dados de censo visual (transectos de 50 x $2 \mathrm{~m}$ ) obtidos nas porções NE, SE e S da costa brasileira revelaram uma distribuição heterogênea entre as localidades, talvez relacionada à pressão de coleta, e uma densidade média de 0.026 ind. $\mathrm{m}^{-2}$. Os resultados sugerem limitação no uso do espaço por H. reidi (consistente com a área vital estimada e com a re-avistagem de exemplares) e que a reprodução ocorre o ano inteiro, embora com possíveis picos. Os componentes da estrutura do habitat mais freqüentemente utilizados como pontos de ancoragem pelos cavalos-marinhos foram vegetação de mangue, macroalgas, cnidários, capim marinho e briozoários. Recomendações conservacionistas incluem caracterização adicional e mapeamento de habitats; avaliação da disponibilidade e condição dos microhabitats em áreas selecionadas, e estudos sobre rotas de dispersão durante as fases iniciais de vida.

Key words: Longsnout seahorse, Mangrove, Habitat use, Population structure, Brazil, Conservation.

\section{Introduction}

Seahorses (genus Hippocampus) are threatened fishes due to declining populations (Martin-Smith \& Vincent, 2006). They are among the many genera whose life histories might render them vulnerable to overfishing or other disruptions such as habitat damage, and are generally characterized by a sparse distribution, low mobility, small home ranges, low fecundity, lengthy parental care, and mate fidelity. In addition the male seahorse, rather than the female becomes pregnant (Foster \& Vincent, 2004).

Nevertheless, different species of seahorses are harvested on a large-scale, and traded by at least 77 nations, in high volumes and different product forms, and degradation of reefs,

\footnotetext{
*Universidade Federal da Paraíba, CCEN, Departamento de Sistemática e Ecologia, Campus I, 58059-900 João Pessoa, PB, Brazil. ierecerosa@yahoo.com.br

**Universidade Federal Fluminense - Departamento de Biologia Marinha, Campus Valonguinho

***Instituto Brasileiro do Meio Ambiente e dos Recursos Naturais Renováveis - IBAMA/Coordenação Geral de Gestão de Recursos Pesqueiros / Diretoria de Fauna e Recursos Pesqueiros

****Universidade Federal de Pernambuco, Centro de Tecnologia e Geociências, Departamento de Oceanografia

*****Universidade Federal de Santa Catarina, Centro de Ciências Agrárias, Departamento de Aqüicultura, Curso de Engenharia de Aqüicultura.
} 
mangroves and seagrass beds has contributed to the depletion of their coastal habitats.

Seahorses are weak swimmers that anchor themselves to vegetation or the substrate with a strong prehensile tail, and rarely venture into open water to pursue mobile prey (Kendrick \& Hyndes, 2005), and are generally considered sit-and-wait predators (Lourie et al., 1999; Foster \& Vincent, 2004; Felício et al., 2006).

The need for understanding space use and ecology of seahorses is increasingly acknowledged in response to concern about their conservation status. Currently, 33 species are listed on the IUCN Red List of Threatened Species (IUCN, 2006), and the entire genus Hippocampus has been added to the Appendix II of the Convention on International Trade in Endangered Species of Wild Fauna and Flora (CITES). Nevertheless, only a few seahorse species have been studied in the wild. This lack of information is reflected in the application of the "Data Deficient" category to most species listed by IUCN, including some heavily exploited ones (see Foster \& Vincent, 2004).

This paper provides valuable base line data about the ecology of Hippocampus reidi Ginsburg, 1933 that can be used for 1) prioritising management and monitoring initiatives; 2 ) evaluating currently protected seahorse habitats; 3 ) identifying additional areas for conservation, and 4) assisting countries in ensuring that trade is non-detrimental, as required by the CITES listing.

\section{Materials and Methods}

The longsnout seahorse Hippocampus reidi is distributed from Cape Hatteras, United States to Brazil and the Gulf of Mexico (Lourie et al., 1999). Within Brazil it has been reported from the northern state of Pará (Salgado region) to the southern state of Rio Grande do Sul (Rosa, 2005).

Information on the surveyed localities is given in Fig. 1 and Tables 1-2. Trade data were gathered through field surveys, as described in Rosa (2005) and Rosa et al. (2005). Visual censuses ( $50 \times 2 \mathrm{~m}$ transect) were performed from September/2002 to September/2004 during morning/afternoon periods, through snorkeling or SCUBA diving, following a standard protocol. Additional data were collected from October/2004 to September/2006. Data collected during pilot observations are only cited anecdotally. The following data were recorded underwater: sex, life stage (adult/juvenile), reproductive state (males: brooding/non-brooding - females: mature/immature), height $(\mathrm{cm})$, depth of the location $(\mathrm{m})$, presence of skin filaments and color pattern. Height (HT) was measured following Project Seahorse's protocol (Lourie et al., 1999). Measurements provided for males and females are those of adult specimens.

Male seahorses were identified by the presence of a brood pouch, while sexual maturity was inferred by full development of the pouch. Females were considered as sexually mature based on their size being equivalent to the mature males (Foster \& Vincent, 2004), and on their ovopositor region be- ing swollen and exhibiting a light coloration. Specimens were filmed, photographed and/or marked with a cotton rib around the tail region.

Nonparametric tests were used in all analyses, as the assumptions of parametric tests were not met. Comparisons among groups (height per sex or locality) were made by means of Mann-Whitney U test and Kruskal-Wallis ANOVA (Zar, 1999). The latter was followed by post hoc Mann-Whitney U and had its $p$ values adjusted for multiple comparisons with Bonferroni's correction when significant differences were found, following Green et al. (2000). Due to the low sample size, data collected at Andorinhas, Itaipu, Ubatuba, Mal Cozinhado and Penha were not compared through post-hoc Mann-Whitney test.

\section{Results}

\section{Population data}

Throughout the study, 911 seahorses were recorded: 322 adult males (35.35\%), 283 adult females (31.06\%) and $306 \mathrm{ju}-$ veniles $(33.60 \%)$, resulting in an unbiased sex ratio $\left(1.14: 1-\chi^{2}\right.$ $=5.03 ; \mathrm{p}=0.03)$. Mean height of all sighted specimens was $11.78 \pm 4.06 \mathrm{~cm}$ (range $=2.4-20.0 \mathrm{~cm})$. Significant differences were found in height distribution among localities $(\mathrm{H}(8, \mathrm{n}=$ $873)=393.38, p<0.01)(F i g .2)$. Population data for the 12 surveyed Brazilian localities are summarized in Table 2.

Height of adult specimens ranged from 5.9 to $20.0 \mathrm{~cm}$ (mean $=13.53 \pm 3.38 \mathrm{~cm}$ ), while height of juveniles ranged from 2.4 to $15.2($ mean $=8.35 \pm 2.92 \mathrm{~cm})$. Eighty five newborns $H$. reidi donated by an exporter measured $0.62 \pm 0.01 \mathrm{~cm}$ (range $=0.48$ $0.75 \mathrm{~cm}$ ). When analyzing the height distribution of all adult specimens combined, males were longer $(14.14 \pm 3.11 \mathrm{~cm} ; \mathrm{n}=$ 318 ; range $=6.5-20.0 \mathrm{~cm})$ than females $(12.84 \pm 3.55 \mathrm{~cm} ; \mathrm{n}=$ 282 ; range $=5.9-19.5 \mathrm{~cm})(\mathrm{U}=35043.5 ; \mathrm{p}=0.000)$. Significant differences between localities were found for male $(\mathrm{H}(6, \mathrm{n}=$ $296)=172.65, \mathrm{p}<0.01)$ and female heights $(\mathrm{H}(6, \mathrm{n}=260)=$ $165.90, \mathrm{p}<0.01$ ) (Tables 3-4).

When combining localities, reproductively active specimens of $H$. reidi were found year-round. The number of seahorses with a fully developed pouch, however, peaked from October to February (summer months) in most study sites. Males with a fully developed brooding pouch $(n=161)$ represented $50.0 \%$ of all adult males found, while mature females $(n=110)$ constituted $39.58 \%$ of all adult females. Mean height of brooding males was $15.58 \pm 1.97 \mathrm{~cm}$ (range $=6.5$ $20.0 \mathrm{~cm}$ ), while mean height of non-brooding specimens was $12.69 \pm 3.37 \mathrm{~cm}(6.5-19.0 \mathrm{~cm})$. Mean height of mature females was $14.88 \pm 2.64 \mathrm{~cm}(5.9-19.5 \mathrm{~cm})$, while mean height of immature specimens was $11.50 \pm 3.44 \mathrm{~cm}(6.0-19.0 \mathrm{~cm})$.

\section{Density and habitat associations}

The longsnout seahorse was unequally distributed across localities, with a mean density of 0.026 ind. $\mathrm{m}^{-2}$ (Table 2). Population density generally was lower at localities were trade was recorded $\left(0.013\right.$ ind. $\mathrm{m}^{-2}$; range $0.008-0.021$ ind. $\left.\mathrm{m}^{-2}\right)$ than at nontrade localities (mean $=0.04$ ind. $\mathrm{m}^{-2}$; range $0.033-0.066$ ind. $\mathrm{m}^{-2}$ ). 
Table 1. Characterization of the surveyed localities.

\begin{tabular}{ll}
\hline $\begin{array}{c}\text { Locality } \\
\text { (Brazilian state) }\end{array}$ & Study site characteristics \\
\hline Camurupim and & Both estuaries located in the municipality of Cajueiro da Praia, within the \\
Ubatuba & limits of an Environmental Protected Area. Surveyed area $2.36 \mathrm{~km}^{2}$ at \\
(Piauí) & Ubatuba and $1.49 \mathrm{Km}^{2}$ at Camurupim. Surveyed depths 0.10 to $1.5 \mathrm{~m}$. \\
& Mangrove forest mainly composed by Rhizophora mangle and Laguncularia \\
& racemosa. Underwater vegetation: mostly macroalgae banks (mainly C. \\
& sertularioides, and mixed stands of red and brown algae). Water salinity 37 \\
& to 44 at Camurupim, 38 to 42 at the Ubatuba. Main economic activities \\
& recorded: commercial fisheries and shrimp farming.
\end{tabular}

Pacoti (Ceará) The estuary is located between Fortaleza and Euzébio $\left(03^{\circ} 53^{\prime 2} 24^{\prime \prime S}\right.$ $\left.38^{\circ} 27^{\prime} 02^{\prime \prime} \mathrm{W}\right)$. Its estimated extension up to the Aquiraz Municipality is $25 \mathrm{~km}$. Encompasses an environmental protected area, and nearly 160 ha of mangrove forest. Impacted by urbanization, deforestation and artisanal fisheries. Maximum surveyed depth: $1.5 \mathrm{~m}$.

Mal Cozinhado Located at the Cascavel municipality $\left(04^{\circ} 07^{\prime} 59^{\prime \prime} \mathrm{S} 38^{\circ} 14^{\prime} 31^{\prime \prime} \mathrm{W}\right)$, with (Ceará) nearly 26 ha of mangrove forest recorded in 2002. Principal species: $R$. mangle, Avicennia germinans and A. schaueriana, L. racemosa, and Conocarpus erectus. Main sources of impact: urbanization, deforestation and artisanal fisheries. Maximum surveyed depth: $1 \mathrm{~m}$.

Tubarão and Both located in the municipality of Macau. The Tubarão estuary is $18.93 \mathrm{~km}^{2}$ Casqueira in area, while Casqueira is approximately $12.2 \mathrm{~km}^{2}$. Since 2003, the Tubarão (Rio Grande do is part of an Environmental Protected Area. Mangrove forest principally Norte) formed by $R$. mangle, L. racemosa and A. shaueriana; underwater vegetation mostly consisting of macroalgae banks (mainly Caulerpa prolifera, $C$. sertularioides, and mixed stands of red and brown algae) and seagrass beds (mainly Halodule wrightii). Water salinity 47 to 50 ; depth 0.10 to $6.0 \mathrm{~m}$. Main sources of impact: shrimp farming and fisheries

Components of habitat structure used by H. reidi as holdfasts

Seahorse trade

Caulerpa sertularioides; Gracilaria, Up to 2003, roots of Rhizophora, Laguncularia; $\quad$ harvesting for fallen branches, shell (Crassostrea rhizophorae) and muddy substrate the aquarium trade. Currently: harvesting for the dried trade.

Caulerpa, Dictyotaceae, Acanthophora, roots of Laguncularia, Rhizophora and Avicennia, fallen branches, $C$. rhizophorae and muddy substrate

Traditional harvesting site for the

Unidentified macroalgae, roots of Laguncularia and Conocarpus, fallen branches and muddy substrate aquarium trade

No trade recorded

Caulerpa spp., Acanthophora, Ulva, Codium sp, Dictyota, Hypnea, unidentified Chlorophyta and Phaeophyta, Halodule wrightii, Halophila; roots of Rhizophora and Avicennia, Porifera, Carijoa, $C$. rhizophorae, Cirrepedia, Ascidia nigra and one unidentified tunicate; muddy substrate

Mamanguape Nearly $24 \mathrm{~km}$ long from east to west, this estuary is part of an environmental (Paraíba) protected area $\left(06^{\circ} 43^{\prime}-06^{\circ} 51^{\prime} \mathrm{S}, 35^{\circ} 07^{\prime}-34^{\circ} 54^{\prime} \mathrm{W}\right)$, and includes ca. 6.000 ha of mangrove forest (mainly: R. mangle, A. germinans, A. schaueriana, $L$. racemosa and Conocarpus erectus). Salinity 17 to 39; depth 0.01 to $2 \mathrm{~m}$. Main sources of impact recorded: sugar cane cultivation, artisanal fisheries, and shrimp farming.

Itapessoca and The Itapessoca and Ariquindá estuaries are located at Goiana and Tamandaré Sargassum, Caulerpa, Hypnea, Ariquindá municipalities, respectively. Itapessoca is $39.98 \mathrm{~km}^{2}$ in area, while Ariquindá (Pernambuco) is approximately $2.37 \mathrm{~km}^{2}$. Mangrove forest mainly formed by $R$. mangle, $A$. shaueriana and L. racemosa. Surveyed depths 0.15 to $3 \mathrm{~m}$. Main recorded sources of impact at Itapessoca: shrimp farming, blast fishing and shrimp trawling; at Ariquindá: tourism (increased recreational boat traffic).

Andorinhas and Costão das Andorinhas and Lagoa de Itaipu are located between the Itaipu coordinates $22^{\circ} 56^{\prime} \mathrm{S} 43^{\circ} 00^{\prime} \mathrm{W}$ and $22^{\circ} 58^{\prime} \mathrm{S} 43^{\circ} 04^{\prime} \mathrm{W}$. Itaipu is a $3.5 \mathrm{~km}$

(Rio de Janeiro) semi-circular reflective sandy beach, flanked north by Costão das Andorinhas, and south by Ponta da Galheta. Maximum surveyed depth:3,5m.

Penha Beach Penha municipality $\left(26^{\circ} 46.8^{\prime} \mathrm{S} 48^{\circ} 36.2^{\prime} \mathrm{W}\right)$ is characterized by the presence (Santa Catarina) of aquaculture ventures . Maximum surveyed depth: $6 \mathrm{~m}$.

Acanthophora, roots of Rhizophora and Avicennia, C. rhizophorae, fallen branches and muddy substrate

Dictyopteris, unidentified macroalgae, branches/logs, roots, leaves and propagules of Rhizophora, Porifera, Carijoa, C. rhizophorae, unidentified gastropod shell, muddy substrate Codium, Ulva spp., Schizoporella spp., No trade
Branchiomma serratibranchis; Styela recorded plicata; rocky/ sandy bottom.

Caulerpa, Gracilaria, Sargassum

Harvesting (dried trade)

Harvesting for aquarium trade at Itapessoca, while at Ariquindá both harvesting and ade recorded.

Harvesting and live trade until 2002.

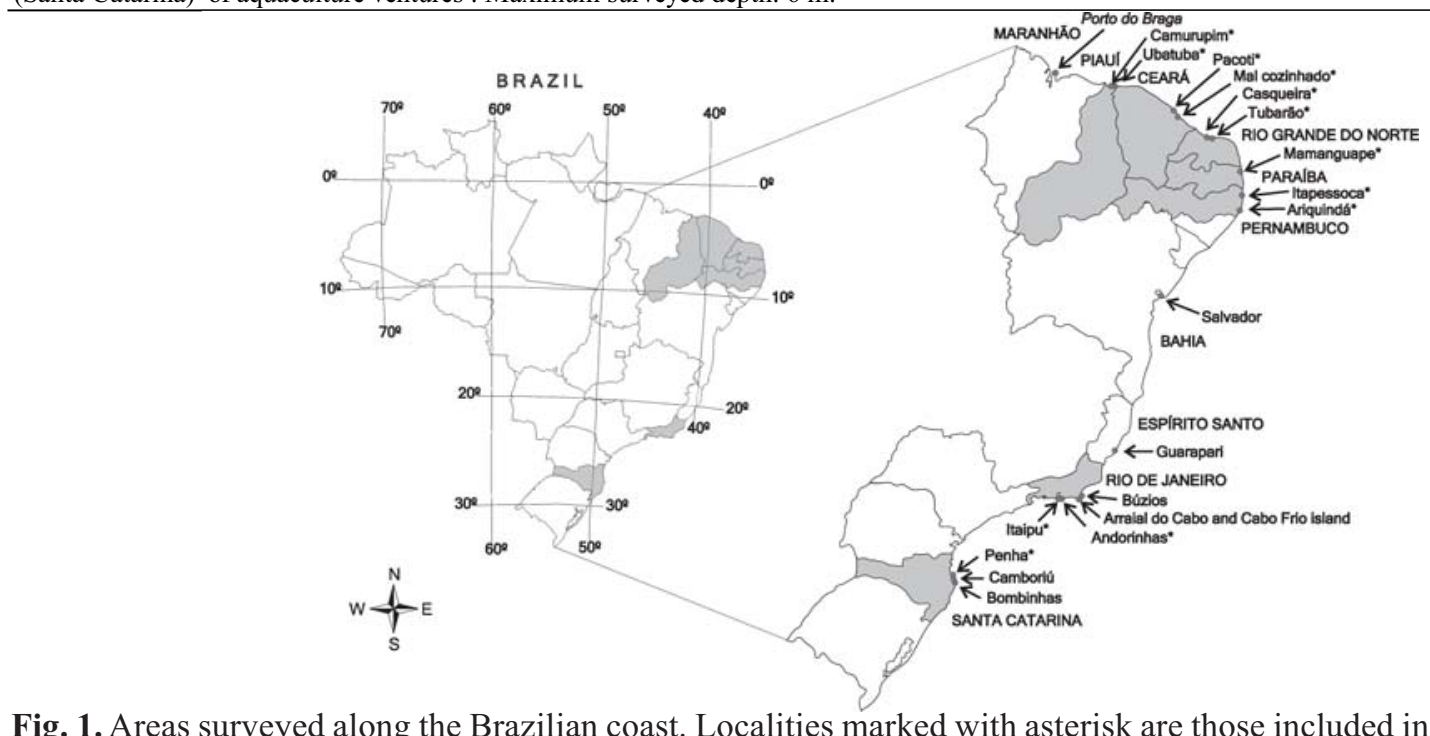

No trade recorded

Fig. 1. Areas surveyed along the Brazilian coast. Localities marked with asterisk are those included in data analyses. 
Table 2. Population data used by Hippocampus reidi in 12 Brazilian localities. Means \pm confidence interval (range); * only one specimen sighted; $\uparrow$ Not compared, due to low sample size.

\begin{tabular}{|c|c|c|c|c|c|c|c|c|c|c|c|c|}
\hline $\begin{array}{c}\text { Surveyed } \\
\text { locality } \\
\text { (Brazilian } \\
\text { state) }\end{array}$ & $\begin{array}{c}\mathrm{N}^{\mathrm{o}} \\
\text { Seahorses } \\
\text { sighted } \\
\left(\mathrm{N}^{\mathrm{o}}\right. \\
\text { transects) }\end{array}$ & $\begin{array}{c}\text { Mean } \\
\text { density } \\
\text { (ind. } \mathrm{m}^{-2} \text { ) }\end{array}$ & $\begin{array}{l}\text { Sex ratio } \\
\text { (M:F) }\end{array}$ & $\begin{array}{l}\text { Height } \\
(\mathrm{cm})\end{array}$ & $\begin{array}{l}\text { Height of } \\
\text { smallest } \\
\text { male with } \\
\text { a pouch } \\
(\mathrm{cm})\end{array}$ & $\begin{array}{l}\text { Height of } \\
\text { non- } \\
\text { brooding } \\
\text { males }\end{array}$ & $\begin{array}{l}\text { Height of } \\
\text { immature } \\
\text { females }\end{array}$ & $\begin{array}{c}\text { Height of } \\
\text { brooding } \\
\text { males }\end{array}$ & $\begin{array}{l}\text { Height of } \\
\text { mature } \\
\text { females }\end{array}$ & $\begin{array}{l}\text { U tests: } \\
\text { Male X } \\
\text { Female }\end{array}$ & $\begin{array}{c}\text { U tests: } \\
\text { Brooding } \\
\text { males x non } \\
\text { brooding } \\
\text { males }\end{array}$ & $\begin{array}{c}\text { U tests: } \\
\text { mature } \\
\text { females } \mathrm{x} \\
\text { immature } \\
\text { females }\end{array}$ \\
\hline $\begin{array}{l}\text { Camurupim } \\
\text { (Piauí) }\end{array}$ & $125(47)$ & $\begin{array}{c}0.0266 \pm \\
0.0081 \\
(0-0.14)\end{array}$ & $\begin{array}{c}2.48: 1 \\
\left(\chi^{2}=28.2\right. \\
\mathrm{p}=0.43)\end{array}$ & $\begin{array}{c}12.6 \\
(3.0-17.5)\end{array}$ & 11.0 & $\begin{array}{c}14.7 \pm 0.6 \\
(11.0-17.0)\end{array}$ & $\begin{array}{c}13.9 \pm 0.5 \\
(6.5-17.0)\end{array}$ & $\begin{array}{c}14.8 \pm 0.5 \\
(12.5- \\
17.5)\end{array}$ & $\begin{array}{c}15.3 \pm 5.02 \\
(13.0- \\
16.5)\end{array}$ & $\begin{array}{c}\mathrm{U}(58.24) \\
=474.0 \\
\mathrm{p}=0.08\end{array}$ & $\begin{array}{c}\mathrm{U}(33.25)= \\
357.5 ; \\
\mathrm{p}=0.81\end{array}$ & $\begin{array}{c}\mathrm{U}(3.21)= \\
19.0 \\
\mathrm{p}=0.32\end{array}$ \\
\hline $\begin{array}{l}\text { Ubatuba } \\
\text { (Piauí) }\end{array}$ & $9(18)$ & $\begin{array}{c}0.0050 \pm \\
0.0040 \\
(0-0.03)\end{array}$ & $\begin{array}{c}0.5: 1 \\
\left(\chi^{2}=1.33\right. \\
\mathrm{p}=0.25)\end{array}$ & $\begin{array}{c}13.83 \\
(7.5-17.0)\end{array}$ & 13.0 & $13.0^{*}$ & $\begin{array}{c}15.5 \pm 6.4 \\
(13.5-16.0)\end{array}$ & $13.0 *$ & $15.0 ; 17.0$ & $\dagger$ & $\dagger$ & $\dagger$ \\
\hline $\begin{array}{l}\text { Pacoti } \\
\text { (Ceará) }\end{array}$ & $169(80)$ & $\begin{array}{c}0.021 \pm \\
0.0068 \\
(0-0.17)\end{array}$ & $\begin{array}{c}0.88: 1 \\
\left(\chi^{2}=1.2\right. \\
p=0.27)\end{array}$ & $\begin{array}{c}7.6 \\
(3.4-16.4)\end{array}$ & 4.2 & $\begin{array}{c}8.5 \pm 0.3 \\
(4.2-13.5)\end{array}$ & $\begin{array}{c}7.8 \pm 0.3 \\
(4.0-11.5)\end{array}$ & $\begin{array}{c}11.6 \pm 2.8 \\
(6.5-16.4)\end{array}$ & $\begin{array}{l}7.1 \pm 1.05 \\
(5.9-9.2)\end{array}$ & $\begin{array}{c}\mathrm{U}(63.72) \\
=1376.5 \\
\mathrm{p}<0.01\end{array}$ & $\begin{array}{c}\mathrm{U}(7.56)= \\
61.5 ; \\
\mathrm{p}<0.01\end{array}$ & $\begin{array}{c}\mathrm{U}(7.65)= \\
143.5 \\
\mathrm{p}=0.11\end{array}$ \\
\hline $\begin{array}{c}\text { Mal } \\
\text { Cozinhado } \\
\text { (Ceará) }\end{array}$ & $62(19)$ & $\begin{array}{c}0.0326 \pm \\
0.0229 \\
(0-0.15)\end{array}$ & $\begin{array}{c}0.25: 1 \\
\left(\chi^{2}=7.2\right. \\
\mathrm{p}<0.01)\end{array}$ & $\begin{array}{c}6.1 \\
(2.4-13.0)\end{array}$ & 3.5 & $3.5^{*}$ & $\begin{array}{c}9.2 \pm 1.04 \\
(3.0-11.3)\end{array}$ & $11.0^{*}$ & $8.0^{*}$ & $\dagger$ & $\dagger$ & $\dagger$ \\
\hline $\begin{array}{c}\text { Tubarão } \\
\text { (Rio Grande } \\
\text { do Norte) }\end{array}$ & $134(26)$ & $\begin{array}{c}0.066 \pm \\
0.044 \\
(0-0.15)\end{array}$ & $\begin{array}{c}1.66: 1 \\
\left(\chi^{2}=6.96\right. \\
\mathrm{p}<0.01)\end{array}$ & $\begin{array}{c}13.5 \\
(5.5-19.5)\end{array}$ & 10.5 & $\begin{array}{c}15.0 \pm 0.5 \\
(10.5-17.2)\end{array}$ & $\begin{array}{c}15.7 \pm 1.4 \\
(13.5-19.0)\end{array}$ & $\begin{array}{c}15.9 \pm 0.5 \\
(13.5- \\
18.0)\end{array}$ & $\begin{array}{c}16.4 \pm 0.7 \\
(10.0- \\
19.5)\end{array}$ & $\begin{array}{l}\mathrm{U}(50.33) \\
=667.5 \\
\mathrm{p}=0.14\end{array}$ & $\begin{array}{c}\mathrm{U}(28.22)= \\
179.5 \\
\mathrm{p}=0.01\end{array}$ & $\begin{array}{c}\mathrm{U}(22.11) \\
=88.0 \\
\mathrm{p}=0.21\end{array}$ \\
\hline $\begin{array}{c}\text { Casqueira } \\
\text { (Rio Grande } \\
\text { do Norte) }\end{array}$ & $198(56)$ & $\begin{array}{c}0.055 \pm \\
0.014 \\
(0.04- \\
0.08)\end{array}$ & $\begin{array}{c}1: 1 \\
\left(\chi^{2}=0.01\right. \\
\mathrm{p}=0.91)\end{array}$ & $\begin{array}{c}14.2 \\
(4.5-20.0)\end{array}$ & 11.0 & $\begin{array}{c}15.6 \pm 0.5 \\
(11.0-19.0)\end{array}$ & $\begin{array}{l}12.8 \pm 0.8 \\
(10-16.5)\end{array}$ & $\begin{array}{c}16.6 \pm 0.4 \\
(14.0- \\
20.0)\end{array}$ & $\begin{array}{c}15.5 \pm 0.4 \\
(10.0- \\
18.0)\end{array}$ & $\begin{array}{c}\mathrm{U}(82.83) \\
=1894.0 \\
\mathrm{p}<0.01\end{array}$ & $\begin{array}{c}\mathrm{U}(59.23)= \\
401.0 \\
\mathrm{p}<0.01\end{array}$ & $\begin{array}{l}\mathrm{U}(54.29) \\
=232.0 \\
\mathrm{p}<0.01\end{array}$ \\
\hline $\begin{array}{l}\text { Mamanguape } \\
\text { (Paraíba) }\end{array}$ & $41(47)$ & $\begin{array}{c}0.0087 \pm \\
0.0024 \\
(0-0.04)\end{array}$ & $\begin{array}{c}1.14: 1 \\
\left(\chi^{2}=0.62\right. \\
p=0.43)\end{array}$ & $\begin{array}{c}13.0 \\
(5.0-18.0)\end{array}$ & 11.0 & $\begin{array}{c}13.25 \pm 2.4 \\
(11.0-15.0)\end{array}$ & $\begin{array}{c}14.0 \pm 2.7 \\
(11.0-17.0)\end{array}$ & $\begin{array}{c}14.9 \pm 1.1 \\
(12.0- \\
18.0)\end{array}$ & $\begin{array}{c}14.6 \pm 0.9 \\
(12.0- \\
16.5)\end{array}$ & $\begin{array}{l}\mathrm{U}(13.16) \\
=103.5 \\
\mathrm{p}=0.98\end{array}$ & $\begin{array}{c}\mathrm{U}(9.4)= \\
7.5 ; \\
\mathrm{p}=0.11\end{array}$ & $\begin{array}{c}\mathrm{U}(11.5)= \\
22.0 \\
\mathrm{p}=0.53\end{array}$ \\
\hline $\begin{array}{c}\text { Itapessoca } \\
\text { (Pernambuco) }\end{array}$ & $80(57)$ & $\begin{array}{c}0.014 \pm \\
0.0058 \\
(0-0.11)\end{array}$ & $\begin{array}{c}1.1: 1 \\
\left(\chi^{2}=0.19\right. \\
\mathrm{p}=0.43)\end{array}$ & $\begin{array}{c}12.8 \\
(5.7-17.0)\end{array}$ & 10.4 & $\begin{array}{c}14.5 \pm 0.43 \\
(10.4-16.0)\end{array}$ & $\begin{array}{c}14.9 \pm 0.48 \\
(6.6-17.0)\end{array}$ & $\begin{array}{c}14.7 \pm 0.8 \\
(13.1- \\
15.8)\end{array}$ & $\begin{array}{c}14.8 \pm 1.04 \\
(13.7- \\
16.0)\end{array}$ & $\begin{array}{c}\mathrm{U}(22.20)= \\
193.5 \\
\mathrm{p}=0.5\end{array}$ & $\begin{array}{c}\mathrm{U}(8.14)= \\
49.0 \\
\mathrm{p}=0.63\end{array}$ & $\begin{array}{c}\mathrm{U}(5.15)= \\
36.0 \\
\mathrm{p}=0.89\end{array}$ \\
\hline $\begin{array}{c}\text { Ariquindá } \\
\text { (Pernambuco) }\end{array}$ & $42(53)$ & $\begin{array}{c}0.0079 \pm \\
0.0036 \\
(0-0.06)\end{array}$ & $\begin{array}{c}0.92: 1 \\
\left(\chi^{2}=0.08\right. \\
\mathrm{p}=0.77)\end{array}$ & $\begin{array}{c}14.0 \\
(6.0-18.0)\end{array}$ & 12.0 & $\begin{array}{c}16.6 \pm 0.8 \\
(12.0-18.0)\end{array}$ & $\begin{array}{c}15.2 \pm 0.7 \\
(6.2-16.8)\end{array}$ & $\begin{array}{c}16.1 \pm 2.36 \\
(15.1- \\
17.0)\end{array}$ & $\begin{array}{c}14.1 \pm \\
4.17 \\
(12.8- \\
16.0)\end{array}$ & $\begin{array}{c}\mathrm{U}(12.13)= \\
22.0 \\
\mathrm{p}<0.01\end{array}$ & $\begin{array}{c}\mathrm{U}(3.9)= \\
8.5 ; \mathrm{p}=0.35\end{array}$ & $\begin{array}{c}\mathrm{U}(3.10)= \\
8.0 \\
\mathrm{p}=0.24\end{array}$ \\
\hline $\begin{array}{l}\text { Andorinhas } \\
\text { (Rio de } \\
\text { Janeiro) }\end{array}$ & 19 (19) & $\begin{array}{c}0.01 \pm \\
0.006 \\
(0-0.05)\end{array}$ & $\begin{array}{c}5: 1 \\
\left(\chi^{2}=5.33\right. \\
\mathrm{p}=0.02)\end{array}$ & $\begin{array}{c}9.3 \\
(5.5-13.9)\end{array}$ & .5 & $\begin{array}{c}12 \pm 0.7 \\
(7.5-12.3)\end{array}$ & $\begin{array}{c}\text { No } \\
\text { specimen } \\
\text { sighted }\end{array}$ & $11.6 ; 13.7$ & $13.9^{*}$ & $\dagger$ & $\dagger$ & $\dagger$ \\
\hline $\begin{array}{l}\text { Itaipu } \\
\text { (Rio de } \\
\text { Janeiro) }\end{array}$ & $27(6)$ & $\begin{array}{c}0.045 \pm \\
0.029 \\
(0-0.08)\end{array}$ & $\begin{array}{c}1.4: 1 \\
\left(\chi^{2}=1.45\right. \\
\mathrm{p}=0.23)\end{array}$ & $\begin{array}{c}13.9 \\
(5.5-19.0)\end{array}$ & 9.1 & $\begin{array}{c}14.7 \pm 3.4 \\
(9.1-16.1)\end{array}$ & $\begin{array}{r}13.9 \\
(5.5-\end{array}$ & $\begin{array}{c}16.0 \pm 1.3 \\
(12.6- \\
19.0)\end{array}$ & $\begin{array}{c}13.9 \pm 2.5 \\
(12.8- \\
15.0)\end{array}$ & $\begin{array}{c}\mathrm{U}(13.9)= \\
24.5 ; \\
\mathrm{p}=0.02\end{array}$ & $\begin{array}{c}\mathrm{U}(10.3)= \\
8.0 ; \mathrm{p}=0.24\end{array}$ & $\begin{array}{c}\mathrm{U}(3.6)= \\
8.0 ; \\
\mathrm{p}=0.79\end{array}$ \\
\hline $\begin{array}{c}\text { Penha } \\
\text { (Santa } \\
\text { Catarina) }\end{array}$ & $5(22)$ & $\begin{array}{c}0.0023 \pm \\
0.0018 \\
(0-0.01)\end{array}$ & - & $\begin{array}{c}8.0 \\
(3.5-11.5)\end{array}$ & 8.0 & $\begin{array}{c}\text { No } \\
\text { specimen } \\
\text { sighted } \\
\end{array}$ & $\begin{array}{c}\text { No } \\
\text { specimen } \\
\text { sighted } \\
\end{array}$ & $\begin{array}{c}\text { No } \\
\text { specimen } \\
\text { sighted }\end{array}$ & $\begin{array}{c}\text { No } \\
\text { specimen } \\
\text { sighted }\end{array}$ & $\dagger$ & $\dagger$ & $\dagger$ \\
\hline
\end{tabular}

As shown on Table 6, specimens of $H$. reidi were most commonly found at lower depths (mean $=0.39 \pm 0.04 \mathrm{~m} ; \mathrm{n}=$ 903 recorded). Adult specimens were most frequently sighted clinging to mangrove trees roots (Laguncularia racemosa, $22.46 \%$, and Rhizophora mangle, $17.66 \%$ ), fallen branches of mangrove plants $(16.47 \%)$, macroalgae $(11.98 \%)$ and the muddy substrate (11.68\%). Young $H$. reidi used mainly mangrove roots (Laguncularia racemosa, 22.17\%, and Rhizophora mangle, 9.91\%), fallen branches of mangrove plants $(19.34 \%)$, macroalgae (16.04\%) and the muddy substrate $(11.32 \%)$ as anchoring points (Table 1). During a pilot survey, one specimen was sighted grasping Millepora $\mathrm{sp.}$ and gorgonian skeletons.

Most specimens sighted were solitary $(73.7 \%$ of sightings, $\mathrm{n}=671$ ), however 102 groupings (Table 4), mostly pairs $(77.5 \% ; \mathrm{n}=79)$ were recorded. 42 male-female interactions were considered as putative partners. Groups of three individuals were sighted 19 times, groups of four twice and groups of 5 and 7 once. Distance between sighted individuals ranged from zero (specimens sharing the same holdfast) to $50 \mathrm{~m}$. Re-

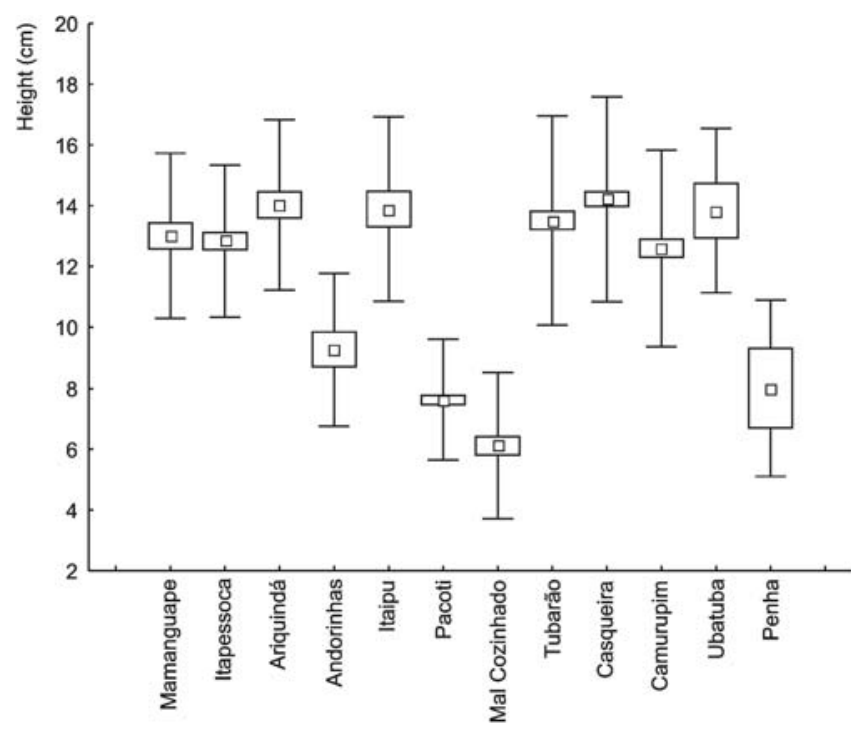

Fig. 2. Height distribution of Hippocampus reidi at 12 Brazilian localities. $\square$ Mean, $\square \pm$ SE, $工 \pm$ SD. 
Table 3. Results of the post-hoc Mann-Whitney test (with the $\mathrm{p}$ values adjusted by the Bonferroni's correction) performed on the heights of male specimens of Hippocampus reidi recorded at seven Brazilian localities.

\begin{tabular}{cccccccc}
\hline Localities & Ariquindá & Camurupim & Casqueira & Itapessoca & & Pamanguape & Tubarão \\
\hline Ariquindá & $\mathrm{X}$ & & & & & & \\
Camurupim & 0.003 & $\mathrm{X}$ & & & & & \\
Casqueira & n.s. & 0.000 & $\mathrm{X}$ & & & & \\
Itapessoca & 0.000 & n.s. & 0.000 & $\mathrm{X}$ & & & \\
Mamanguape & 0.011 & n.s. & 0.011 & & $\mathrm{X}$ & $\mathrm{X}$ & \\
Pacoti & 0.000 & 0.000 & 0.000 & 0.000 & 0.000 & 0.000 & $\mathrm{X}$ \\
Tubarão & 0.285 & 0.140 & 0.271 & 0.123 & 0.709 & 0.000 \\
\hline
\end{tabular}

Table 4. Results of the post-hoc Mann-Whitney test (with the $\mathrm{p}$ values adjusted by the Bonferroni's correction) performed on the heights of female specimens of Hippocampus reidi recorded at seven Brazilian localities.

\begin{tabular}{cccccccc}
\hline Localities & Ariquindá & Camurupim & Casqueira & Itapessoca & Mamanguape & Pacoti & Tubarão \\
\hline Ariquindá & $\mathrm{X}$ & & & & & & \\
Camurupim & n.s & $\mathrm{X}$ & & & & & \\
Casqueira & n.s & n.s. & $\mathrm{X}$ & & & & \\
Itapessoca & n.s. & 0.092 & n.s. & $\mathrm{X}$ & & & \\
Mamanguape & n.s. & n.s. & n.s. & n.s. & $\mathrm{X}$ & & \\
Pacoti & 0.000 & 0.000 & 0.000 & 0.000 & 0.000 & $\mathrm{X}$ & \\
Tubarão & 0.732 & 0.001 & 0.020 & 0.164 & 0.062 & 0.000 & $\mathrm{X}$ \\
\hline
\end{tabular}

Table 5. Social associations and records of reproductive behavior in Hippocampus reidi at 12 Brazilian localities (means reported with confidence interval and range). ?=ongoing research (state of Piauí) or no data collected (elsewhere).

\begin{tabular}{|c|c|c|c|c|c|c|c|}
\hline $\begin{array}{c}\text { Locality } \\
\text { (Brazilian state) }\end{array}$ & $\begin{array}{l}\text { Specimens sighted } \\
\text { solitary }(\mathrm{S}) \text { or in } \\
\text { groups }(\mathrm{G})\end{array}$ & $\begin{array}{l}\% \text { of sightings } \\
\text { of seahorses } \\
\text { (S) }\end{array}$ & $\begin{array}{l}\mathrm{N} \text { specimens } \\
\text { per group }\end{array}$ & $\begin{array}{l}\text { Mean distance } \\
\text { between } \\
\text { individuals }(\mathrm{m})\end{array}$ & $\begin{array}{l}\text { Re-sighting of } \\
\text { individuals }\end{array}$ & $\begin{array}{l}\text { Record of } \\
\text { courtship } \\
\text { behavior }\end{array}$ & $\begin{array}{l}\text { Months where } \\
\text { brooding males } \\
\text { were sighted }\end{array}$ \\
\hline $\begin{array}{l}\text { Camurupim } \\
\text { (Piauí) }\end{array}$ & $\mathrm{S} / \mathrm{G}$ & 75.5 & $2,3,5$ & $\begin{array}{c}6.47 \pm 1.70(0- \\
18.8)\end{array}$ & Yes & No & ? \\
\hline Ubatuba (Piauí) & $\mathrm{S} / \mathrm{G}$ & 77.8 & 2 & ? & No & No & ? \\
\hline Pacoti (Ceará) & $\mathrm{S} / \mathrm{G}$ & 74.6 & $2-4,7$ & $7.11 \pm 1.65(0-41)$ & $?$ & No & October-December \\
\hline $\begin{array}{l}\text { Mal Cozinhado } \\
\text { (Ceará) }\end{array}$ & $\mathrm{S} / \mathrm{G}$ & 75.8 & $2-3$ & $\begin{array}{c}3.42 \pm 1.27(0- \\
23.5)\end{array}$ & $?$ & No & November \\
\hline $\begin{array}{l}\text { Casqueira (Rio } \\
\text { Grande do Norte) }\end{array}$ & $\mathrm{S} / \mathrm{G}$ & 77.3 & $2-3$ & $?$ & Yes & Yes & Year-round \\
\hline $\begin{array}{c}\text { Tubarão (Rio } \\
\text { Grande do Norte) }\end{array}$ & $\mathrm{S} / \mathrm{G}$ & 68.7 & $2-3$ & $?$ & Yes & Yes & Year-round \\
\hline $\begin{array}{l}\text { Mamanguape } \\
\text { (Paraíba) }\end{array}$ & $\mathrm{S} / \mathrm{G}$ & 58.5 & $2-4$ & $?$ & Yes & Yes & Year-round \\
\hline $\begin{array}{c}\text { Itapessoca } \\
\text { (Pernambuco) }\end{array}$ & $\mathrm{S} / \mathrm{G}$ & 85.0 & 2 & $8.60 \pm 2.9(0-38)$ & $?$ & No & Year-round \\
\hline $\begin{array}{c}\text { Ariquindá } \\
\text { (Pernambuco) }\end{array}$ & $\mathrm{S} / \mathrm{G}$ & 71.4 & 2 & $7.36 \pm 5.5(0-50)$ & $?$ & Yes & Year-round \\
\hline $\begin{array}{l}\text { Andorinhas (Rio } \\
\text { de Janeiro) }\end{array}$ & $\mathrm{S} / \mathrm{G}$ & 68.4 & 2 & $\begin{array}{c}3.81 \pm 2.06(0- \\
14.7)\end{array}$ & Yes & No & $\begin{array}{l}\text { August and } \\
\text { November }\end{array}$ \\
\hline $\begin{array}{l}\text { Itaipu (Rio de } \\
\text { Janeiro) }\end{array}$ & $\mathrm{S} / \mathrm{G}$ & 77.8 & 2 & $\begin{array}{c}3.42 \pm 2.04(0- \\
17.6)\end{array}$ & Yes & No & $\begin{array}{l}\text { February, June, } \\
\text { August and } \\
\text { December }\end{array}$ \\
\hline $\begin{array}{l}\text { Penha (Santa } \\
\text { Catarina) }\end{array}$ & $\mathrm{S}$ & 100.0 & $\begin{array}{c}\text { No grouping } \\
\text { observed }\end{array}$ & ? & No & No & $?$ \\
\hline
\end{tabular}

sighting of individuals occurred in most surveyed areas (Table 5). Within a year, the maximum period of time between the original sighting and re-sighting of marked specimens was 264 days $($ mean $=69.40 \pm 49.47)$.

Eleven specimens were found with their tail tip clipped, suggesting partial predation by crabs; one specimen was sighted while moving away from a crab (genus Callinectes). Two specimens had anemones attached on the operculum, dorsum and between their eyes; two others were sighted with a hermit crab on their dorsum.

\section{Reproductive behavior}

Mating was only recorded once, at $0.25 \mathrm{~m}$ depth, as fol- lows: one male and one female sharing a holdfast started moving for short distances (less than $1.0 \mathrm{~m}$ ), around their holdfast, parallel to each other, grasping tails as they swirled around, and then freed their tails (during this behavior occasionally the pair grasped the holdfast). The male pumped his tail against the body region (3-5 times consecutively), a behavior that was followed by the pair assuming a pointing position (in which both seahorses pointed their snouts upwards), and repeatedly rising in the water column. Subsequently, eggs were transferred, the female placing her ovipositor inside the male's pouch opening. Immediately after mating, male and female separated, each grasping a holdfast, approximately $1.5 \mathrm{~m}$ apart from each other. The male then 
Table 6. Mean, confidence interval and range of depths (m) at which Hippocampus reidi was sighted in 12 Brazilian localities.

\begin{tabular}{|c|c|c|c|c|c|}
\hline \multirow{3}{*}{ Locality (Brazilian state) } & \multirow[t]{2}{*}{ Juveniles } & \multicolumn{2}{|c|}{ Males } & \multicolumn{2}{|c|}{ Females } \\
\hline & & Brooding & Non-Brooding & Mature & Immature \\
\hline & $0.48 \pm 0.09(0.03-6.0)$ & $0.42 \pm 0.08(0.03-2.70)$ & $0.26 \pm 0.06(0.02-2.50)$ & $0.38 \pm 0.09(0.05-2.19)$ & $0.31 \pm 0.06(0.00-2.50)$ \\
\hline Camurupim (Piauí) & $\begin{array}{c}0.24 \pm 0.07 \\
(0.05-1.50)(\mathrm{n}=45)\end{array}$ & $\begin{array}{c}0.19 \pm 0.04 \\
(0.05-0.60)(\mathrm{n}=33)\end{array}$ & $\begin{array}{c}0.25 \pm 0.12 \\
(0.10-1.10)(\mathrm{n}=24)\end{array}$ & $\begin{array}{c}0.42 \pm 0.27 \\
(0.15-0.60)(\mathrm{n}=3)\end{array}$ & $\begin{array}{c}0.36 \pm 0.17 \\
(0.10-1.30)(\mathrm{n}=20)\end{array}$ \\
\hline Ubatuba (Piauí) & $\begin{array}{c}0.17 \pm 0.03 \\
(0.15-0.20)(\mathrm{n}=3)\end{array}$ & $0.05(\mathrm{n}=1)$ & $0.10(\mathrm{n}=1)$ & $\begin{array}{c}0.15 \pm 0.10 \\
(0.10 ; 0.20)(\mathrm{n}=2)\end{array}$ & $\begin{array}{c}0.40 \pm 0.59 \\
(0.10 ; 0.70)(\mathrm{n}=2)\end{array}$ \\
\hline Pacoti (Ceará) & $\begin{array}{c}0.18 \pm 0.06 \\
(0.03-1.10)(\mathrm{n}=34)\end{array}$ & $\begin{array}{c}0.24 \pm 0.26 \\
(0.03-1.00)(\mathrm{n}=7)\end{array}$ & $\begin{array}{c}0.11 \pm 0.03 \\
(0.02-0.50)(\mathrm{n}=56)\end{array}$ & $\begin{array}{c}0.05 \pm 0.00 \\
(0.05 \text {-all of them })(n=7)\end{array}$ & $\begin{array}{c}0.20 \pm 0.05 \\
(0.03-1.30)(\mathrm{n}=65)\end{array}$ \\
\hline Mal Cozinhado (Ceará) & $\begin{array}{c}0.20 \pm 0.04 \\
(0.03-0.6)(\mathrm{n}=52)\end{array}$ & $\begin{array}{c}0.8 \\
(\mathrm{n}=1)\end{array}$ & $\begin{array}{c}0.05 \\
(\mathrm{n}=1)\end{array}$ & $\begin{array}{c}0.1 \\
(\mathrm{n}=1)\end{array}$ & $\begin{array}{c}0.10 \pm 0.04 \\
(0.03-0.2)(\mathrm{n}=7)\end{array}$ \\
\hline Casqueira (Rio Grande do Norte) & $\begin{array}{c}0.91 \pm 0.27 \\
(0.1-3.0)(n=33)\end{array}$ & $\begin{array}{c}0.55 \pm 0.15 \\
(0.1-1.7)(\mathrm{n}=59)\end{array}$ & $\begin{array}{c}0.39 \pm 0.20 \\
(0.1-1.6)(\mathrm{n}=23)\end{array}$ & $\begin{array}{c}0.46 \pm 0.15 \\
(0.1-1.8)(\mathrm{n}=54)\end{array}$ & $\begin{array}{c}0.39 \pm 0.24 \\
(0.1-2.5)(\mathrm{n}=29)\end{array}$ \\
\hline Tubarão (Rio Grande do Norte) & $\begin{array}{c}0.15 \pm 0.03 \\
(0.1-0.5)(\mathrm{n}=51)\end{array}$ & $\begin{array}{c}0.15 \pm 0.05 \\
(0.1-0.7)(\mathrm{n}=28)\end{array}$ & $\begin{array}{c}0.14 \pm 0.03 \\
(0.1-0.4)(n=22)\end{array}$ & $\begin{array}{c}0.14 \pm 0.04 \\
(0.1-0.5)(\mathrm{n}=22)\end{array}$ & $\begin{array}{c}0.15 \pm 0.05 \\
(0.1-0.3)(\mathrm{n}=11)\end{array}$ \\
\hline Mamanguape (Paraíba) & $\begin{array}{c}0.40 \pm 0.19 \\
(0.08-1.00)(\mathrm{n}=12)\end{array}$ & $\begin{array}{c}0.27 \pm 0.13 \\
(0.10-0.60)(\mathrm{n}=4)\end{array}$ & $\begin{array}{c}0.45 \pm 0.55 \\
(0.06-1.00)(\mathrm{n}=9)\end{array}$ & $\begin{array}{c}0.24 \pm 0.15 \\
(0.05-0.70)(\mathrm{n}=11)\end{array}$ & $\begin{array}{c}0.93 \pm 0.65 \\
(0.22-1.50)(\mathrm{n}=5)\end{array}$ \\
\hline Itapessoca (Pernambuco) & $\begin{array}{c}0.44 \pm 0.13 \\
(0.05-1.8)(\mathrm{n}=38)\end{array}$ & $\begin{array}{c}0.74 \pm 0.65 \\
(0.04-2.7)(\mathrm{n}=8)\end{array}$ & $\begin{array}{c}0.41 \pm 0.23 \\
(0.06-1.5)(\mathrm{n}=14)\end{array}$ & $\begin{array}{c}0.8 \pm 0.38 \\
(0.08-1.10)(\mathrm{n}=5)\end{array}$ & $\begin{array}{c}0.42 \pm 0.19 \\
(0.0-1.10)(\mathrm{n}=15)\end{array}$ \\
\hline Ariquindá (Pernambuco) & $\begin{array}{c}0.17 \pm 0.03 \\
(0.09-0.35)(\mathrm{n}=24)\end{array}$ & $\begin{array}{c}0.17 \pm 0.10 \\
(0.10-0.27)(\mathrm{n}=3)\end{array}$ & $\begin{array}{c}0.14 \pm 0.05 \\
(0.05-0.25)(\mathrm{n}=9)\end{array}$ & $\begin{array}{c}0.23 \pm 0.13 \\
(0.10-0.33)(\mathrm{n}=3)\end{array}$ & $\begin{array}{c}0.18 \pm 0.08 \\
(0.04-0.42)(\mathrm{n}=10)\end{array}$ \\
\hline Andorinhas (Rio de Janeiro) & $\begin{array}{c}2.46 \pm 0.39 \\
(1.50-3.50)(\mathrm{n}=13)\end{array}$ & $\begin{array}{c}2.25 \pm 0.49 \\
(2.00 ; 2.50)(\mathrm{n}=2)\end{array}$ & $\begin{array}{c}2.17 \pm 0.33 \\
(2.00-2.5)(\mathrm{n}=3)\end{array}$ & $2.19(\mathrm{n}=1)$ & - \\
\hline Itaipu (Rio de Janeiro) & $\begin{array}{c}0.90 \pm 0.20 \\
(0.50-1.00)(\mathrm{n}=5)\end{array}$ & $\begin{array}{c}0.98 \pm 0.05 \\
(0.75-1.00)(n=10)\end{array}$ & $\begin{array}{c}0.67 \pm 0.33 \\
(0.50-1.00)(\mathrm{n}=3)\end{array}$ & $\begin{array}{c}1.00 \pm 0.00 \\
(1.00 \text {-all of them })(n=3)\end{array}$ & $\begin{array}{c}1.00 \pm 0.22 \\
(0.75-1.50)(\mathrm{n}=6)\end{array}$ \\
\hline Penha (Santa Catarina) & $\begin{array}{c}4.4 \pm 1.7 \\
(2.00-6.00)(\mathrm{n}=5)\end{array}$ & - & - & - & - \\
\hline
\end{tabular}

rocked back and forth for 3 min. Total duration of the observed reproductive behavior was of $25 \mathrm{~min}$. A male observed giving birth (at 11:00 am) pumped the tail against the body region twice, expelling approximately 100 young each time (height of one newborn: $8 \mathrm{~mm}$ ). Dispersal of young was initiated shortly after that.

\section{Discussion}

\section{Population Parameters}

Although seahorse populations commonly have an unbiased sex ratio (Lourie et al., 1999), biased sex ratios have been recorded in some species (e.g. Perante et al., 1998; Teixeira \& Musick, 2001; Bell et al., 2003; Martin-Smith \& Vincent, 2005). The equivalent total number of males and females observed in our study agrees with the results obtained by Dauwe (1992) for $H$. reidi, as well as with studies on some other seahorse species (Vincent \& Sadler, 1995; Bell et al., 2003; Moreau \& Vincent, 2004; Curtis \& Vincent, 2006). The discrepancies found at the Camurupim site may be related to the seahorse harvesting which took place until 2003, whereas at the Mal Cozinhado it reflects the few records of adult males (which were used to estimate the sex ratio).

Combined sightings of brooding males suggest that reproductive period of $H$. reidi extends year-round, a result consistent with the information provided by Vincent (1990), and with the hypothesis that tropical seahorse species may have a lengthier reproductive period than temperate species, extending throughout the whole 12 months (Foster \& Vincent, 2004). A lengthy reproductive period has also been found to pipefishes (e.g. Howard \& Koehn, 1985; Gasparini \& Teireira,
1999; Lyons \& Dunne, 2005).

The smaller proportion of juvenile $H$. reidi found in our study is in line with previous research (e.g. Bell et al., 2003; Moreau \& Vincent, 2004), and is typical of seahorse population assessments (Martin-Smith \& Vincent, 2005). It has been suggested that very young $H$. reidi may spend time in the plankton, and its development and behavior may be pelagic based on the tendency not to use the tail for anchorage (Bull, 2002). Consistent with that information, very young $H$. reidi have been captured in plankton nets (Ekau et al., 2001), and some have been observed dispersing in the water column shortly after being born (this study).

Although greater male height is unusual in seahorses (Foster \& Vincent, 2004), larger males have been observed in $H$. capensis (Bell et al., 2003), H. spinosissimus and $H$. trimaculatus (see Foster \& Vincent, 2004), as well as in $H$. reidi (Dias, 2002; Barros, 2005). Male height was found to be positively related to clutch size in other syngnathid species (Strawn, 1958; Berglund et al., 1986; Masonjones, 1997), however, in $H$. fuscus, $H$. erectus and $H$. whitei, a positive correlation was found between female size and number of offspring (Vincent, 1990; Teixeira \& Musick, 2001; Vincent \& Giles, 2003), a result consistent with studies on two species of pipefish (Berglund et al., 1986, 1988).

In this study, brooding males and mature females were longer than non-brooding males and immature females, respectively. Size differences related to reproduction have been interpreted as perhaps reflecting pairing of large males with larger females (Teixeira \& Musick, 2001), rather than being a direct effect of male size resulting from individuals trying to maximize their reproductive potential (Foster \& Vincent, 2004). 


\section{Reproductive behavior}

Our results in general agree with previous studies, in which courtship and copulation events interactions were recorded in the morning (Vincent, 1995; Grange \& Cretchley, 1995; Vincent \& Sadler, 1995; Masonjones \& Lewis, 1996; Moreau \& Vincent, 2004). Observed reproductive interactions in $H$. reidi are common courtship behavior patterns in seahorses (Vincent, 1995; Grange \& Cretchley, 1995; Vincent \& Sadler, 1995; Woods, 2000; Moreau \& Vincent, 2004). The duration of $H$. reidi's courtship encounters closely approached the mean of 6 min observed for $H$. whitei (Vincent \& Sadler, 1995), and the $5.2 \mathrm{~min}$ mean found for all but the first day of courtship in H. zosterae (Masonjones \& Lewis, 1996); such patterns could represent common courtship behaviour across species.

According to the descriptions provided by Lourie et al. (1999) and Masonjones \& Lewis (2000), the coupling seahorses sighted in our surveys were in the last phases of courtship. These two distinct behavior patterns have been interpreted as meaning readiness to mate (Vincent, 1990, 1994a, 1994b; Masonjones \& Lewis, 1996). The observed slow and repeated body movements just after eggs transfer has been interpreted as an attempt to settle/distribute the eggs in the pouch (Masonjones \& Lewis, 1996; Lourie et al., 1999; Woods, 2000).

Although color changes, which may mean social interaction (Vincent, 1994a), are commonly observed in seahorses' encounters (Vincent, 1995; Vincent \& Sadler, 1995; Woods, 2000; Foster \& Vincent, 2004; Moreau \& Vincent, 2004), there were no conclusive evidences of color changes in the coupling seahorses sighted.

\section{Density and habitat associations}

The longsnout seahorse exhibited a patchy distribution pattern and low densities, a general tendency described to seahorse species (Lourie et al., 1999, 2004; Foster \& Vincent, 2004). Lower mean population densities generally were found at localities where harvesting of seahorses has been recorded. However, the lowest density recorded at the Penha site is possibly related to $H$. reidi approaching the end of its southern Brazilian distribution, an area in which its density is known to be naturally lower (Rosa, 2005).

A previous focal grid study (Dias \& Rosa, 2003) recorded mean densities of 0.51 and 0.006 ind $\mathrm{m}^{-2}$ at a wooden pier and in an estuarine area, respectively. Mean densities in areas where seahorse harvesting occurs were roughly the same as the density the exploited $H$. comes (Perante et al., 2002), but were lower (even among areas without harvesting in Brazil), than those obtained in focal grid studies on $H$. whitei (Vincent \& Sadler, 1995; Vincent et al., 2005), H. breviceps (Moreau \& Vincent, 2004), H. capensis (Bell et al., 2003), and H. guttulatus (Curtis \& Vincent, 2005, 2006).

Densities estimated for seahorses generally are lower in random transect surveys than in focal grids (see Foster \& Vincent, 2004), even if they are unharvested species (e.g. H. capensis, Bell et al., 2003; H. hippocampus and H. guttulatus,
Curtis \& Vincent, 2005; H. abdominalis, Martin-Smith \& Vincent, 2005), probably due to abiotic and biotic conditions (Curtis \& Vincent, 2006), and possibly related to the sparse distribution of seahorse species or sampling constraints. However, as remarked by Foster \& Vincent (2004), at least in some populations of Hippocampus the low densities probably derive from overexploitation. We observed this at some of our study sites, particularly in the state of Ceará, where population density and mean height of adults were significantly lower than at other sites. We recommend further population studies at the Ceará sites where the smallest specimens were found, to evaluate whether that characteristic is related to fishing pressure or not, and to better assess the implications of size distribution in terms of reproductive output.

The holdfasts recorded in our study were consistent with the description provided by Rosa et al. (2002) and Dias \& Rosa (2003) for the species. Our findings clearly indicated the important role played by components of habitat structure (e.g., mangrove vegetation) in maintaining healthy populations of $H$. reidi.

Influence of habitat availability on the spatial distribution and abundance of $H$. reidi was suggested by the use of the locally abundant bryozoans and macroalgae as anchoring points in SE/S localities, and of mangrove roots in NE Brazil. This result is consistent with Curtis \& Vincent (2005), who observed that the amount of total habitat covered by vegetation and sessile invertebrates appeared to influence the distribution and abundance of $H$. guttulatus.

Specimens of $H$. reidi were often found resting their bodies on the substrate, a behavior also described to $H$. capensis (Bell et al., 2003) and H. abdominalis (Martin-Smith \& Vincent, 2005). As inferred for $H$. breviceps by Moreau \& Vincent (2004), specimens encountered on bare substrate may be temporarily exploiting open habitats, be in transit between covered microhabitats or have been displaced from their home ranges by strong wave action during storms.

Some seahorse species are known to grasp artificial structures, such as fishnets and cages or jetty piles (see Foster \& Vincent, 2004). H. reidi is also known to use man-made structures, such as wooden piers (Dias \& Rosa, 2003), and in our study were sighted clinging to fish corral screen and nylon ropes. The use of artificial substrates as holdfasts, although suggesting a relative plasticity by the species in relation to anthropogenic modifications of the coastal environments can also render the seahorses more accessible to direct exploitation, or increase the risk of accidental removal of specimens during haulage of nets or other fishing gear.

Home ranges have been associated with reproductive behavior in some seahorse species (Vincent \& Sadler, 1995; Perante et al., 2002; Bell et al., 2003; Vincent et al., 2005; Curtis \& Vincent, 2006), with the costs of associated with movement being greater than the benefits in terms of survival, growth and reproduction (Vincent et al., 2005). Spatial fidelity to sites and putative pairs were suggested by the resighting of individuals in most surveyed areas, corroborating patterns previously demonstrated in this species (Dauwe, 
1992; Nijhoff, 1993; Rosa et al., 2002).

Hippocampus reidi is known to occur between depths of about $10 \mathrm{~cm}$ (Rosa et al., 2002) and $55 \mathrm{~m}$ Vari (1982), and sightings in shallow water emphasizes its coastal pattern and highlights the importance of coastal habitats, such as mangrove and reefs, for this species and most other seahorse species (see Foster \& Vincent, 2004). However, given that Dauwe (1992) recorded larger individuals of this species in deeper waters, our records of the species at greater depths should be further investigated to evaluate the role played by local habitat structure, ontogeny and/or reproductive stage in the species' vertical distribution.

The seahorses in our study were generally found to be solitary with no other individual within visual range. Similar results were also obtained for $H$. abdominalis (Martin-Smith \& Vincent, 2005). When not solitary, H. reidi specimens were mostly observed in pairs, generally putative partners. Hippocampus reidi pair forming has been previously recorded by Dauwe (1992) and Nijhoff (1993), and groups up to four individuals were recorded by Rosa et al. (2002). As observed in the wild for the socially monogamous species H. whitei (Vincent \& Sadler, 1995), H. comes (Perante et al., 2002) and $H$. capensis, the commonest social group sighted in our study was male/female pair. This result plus the observed site fidelity and small home range, suggests that Hippocampus reidi may follow a monogamous sexual pattern, as is generally found in other species of seahorses (see Foster \& Vincent, 2004).

Given the need to assess the percentage of existing and potential refugia, and the potential impact of harvesting on wild populations of seahorses, conservation recommendations include: 1) further characterization and mapping of seahorse habitats; 2) an evaluation of the extent of habitat that is currently protected; 3 ) identification of suitable habitat network, based on seahorse life history and ontogeny; 4) assessment of availability and condition of microhabitats in selected areas, with and without legal protection; 5) studies on dispersal routes during initial life stages, and 6) ongoing assessment of abundance from fishery-independent surveys; 7) studies focusing on recognized sources of mortality in aquaria (e.g., feeding and nutrition), both to improve survival rates, and to generate data necessary to develop a science-based protocol for sustainable aquaculture of the species.

Seahorses have an important role as flagship species to promote the conservation of marine ecosystems. Furthermore, as remarked by Martin-Smith \& Vincent (2006), together with their syngnathid relatives, seahorses have provided a focus for efforts to ensure sustainable use of marine resources, with new international trade controls via CITES. Given that their populations in the wild are threatened by a number of anthropogenic factors which also threaten less well-known species living in coastal habitats, a continued effort to conserve seahorses and their habitats may thus benefit broader assemblages of marine species.

\section{Acknowledgments}

To PROBIO/MMA/IBRD/GEF/CNPq for financial support. To IBAMA (Instituto Brasileiro do Meio Ambiente e dos Recursos Naturais Renováveis) for providing research permits, and for working in partnership with us. Within IBAMA, special thanks are due to Glaura Maria L. Barros (GEREX-CE), Clerton de Paula Pontes (CEPENE), Luiz Fernando Rodrigues and Ana Maria Torres (CEPSUL), and Carla Marcon (GEREXPB) for their enthusiasm and support throughout the study. To CAPES (Coordenação de Aperfeiçoamento de Pessoal de Nível Superior) for providing a M.Sc. scholarship to A. L. C. Castro and A. C. G. Mai. To CNPq (Conselho Nacional de Desenvolvimento Científico e Tecnológico) for providing a research fellowship to I. L. Rosa, and a M.Sc. scholarship to T. P. R. Oliveira. Thanks are also due to the Universidade Federal da Paraíba (Departamento de Sistemática e Ecologia, Programa de Pós-Graduação em Ciências Biológicas and NEPREMAR) for all their support. Special thanks are due to Arlindo, Pedro Bala, Feliciano José ("Seu Lúcio"), Adrianne Barros, José de Anchieta, Eugênio Pacelli, Aline Diniz, Gabrielle Tenório and Ieda Zaparolli, for the their fundamental support during field work.

\section{Literature Cited}

Barros, A. T. 2005. Taxonomia e análise da estrutura populacional comercializada de cavalos-marinhos (Syngnathidae: Teleostei: Hippocampus) no Brasil. Unpublished Dissertation, Universidade Federal da Paraíba, João Pessoa, 63p.

Bell, E. M., J. F. Lockyear, J. M. McPherson, A. D. Marsden \& A. C. J. Vincent. 2003. The first field studies of an endangered South African seahorse, Hippocampus capensis. Environmental Biology of Fishes, 67:35-46.

Berglund, A., G. Rosenqvist \& I. Svensson. 1986. Mate choice, fecundity and sexual dimorphism in two pipefish species (Syngnathidae). Behavioral Ecology and Sociobiology, 19:307310.

Berglund, A., G. Rosenqvist \& I. Svensson. 1988. Multiple matings and paternal brood care in the pipefish Syngnathus typhle. Oikos, 51:184-188.

Bull, C. 2002. Seahorse Husbandry in Public Aquaria: 2002 Manual, with chapters contributed by members of the Syngnathid Discussion Group. Chicago, John G. Shedd Aquarium, 56p.

Curtis, J. M. R. \& A. C. J. Vincent. 2005. Distribution of sympatric seahorse species along a gradient of habitat complexity in a seagrass-dominated community. Marine Ecology Progress Series, 291:81-91.

Curtis, J. M. R. \& A. C. J. Vincent. 2006. Life history of an unusual marine fish: survival, growth and movement patterns of Hippocampus guttulatus (Cuvier 1829). Journal of Fish Biology, 68:707-733.

Dauwe, B. 1992. Ecology of the seahorse Hippocampus reidi on the Bonaire coral reef (N.A.): habitat, reproduction and community interactions. Unpublished Dissertation, Rijksuniversiteit Groningen, 63p.

Dias, T. L. P. 2002. Ecologia populacional de Hippocampus reidi Ginsburg, 1933 (Teleostei: Syngnathidae) no Estado do Rio 
Grande do Norte, Brasil. Unpublished Dissertation, Universidade Federal da Paraíba, João Pessoa, 77p.

Dias, T. L. P. \& I. L. Rosa. 2003. Habitat preferences of a seahorse species, Hippocampus reidi (Teleostei: Syngnathidae) in Brazil. Aqua Journal of Ichthyology and Aquatic Biology, 6:165-176.

Ekau, W., P. Westhaus-Ekau, S. J. Macêdo \& C. V. Dorrien. 2001. The larval fish fauna of the "Canal de Santa Cruz" estuary in northeast Brazil. Tropical Oceanography, 29:1-12.

Felício, A. K. C., I. L. Rosa, A. Souto \& R. H. A. Freitas. 2006. Feeding behavior of the longsnout seahorse Hippocampus reidi Ginsburg, 1933. Journal of Ethology, 24(3):219-225.

Foster, S. J. \& A. C. J. Vincent. 2004. Life history and ecology of seahorses: implications for conservation and management. Journal of Fish Biology, 65:1-61.

Gasparini, J. L. \& R. L. Teixeira. 1999. Reproductive aspects of the gulf pipefish, Syngnathus scovelli (Teleostei: Syngnathidae), from Southeastern Brazil. Revista Brasileira de Biologia, 59(1):8790.

Ginsburg, I. 1937. Review of seahorses (Hippocampus) found on the coats of the American continents and Europe. In: Proceedings of the United States National Museum, 83(2997):497-594.

Grange, N. \& R. Cretchley. 1995. A preliminary investigation of the reproductive behavior of the Knysna seahorse Hippocampus capensis Boulanger, 1900. Southern African Journal of Aquatic Sciences, 21:103-104.

Green, S. B., N. J. Salkind \& T. M. Akey. 2000. Using SPSS for Windows: analysing and understanding data. New Jersey, Prentice Hall, 430p.

Howard, R. K. \& J. D. Koehn. 1985. Population dynamics and feeding ecology of pipefish (Syngnathidae) associated with eelgrass beds of Western Port, Victoria. Australian Journal of Marine and Freshwater Research, 36(3):361-370

IUCN. 2006. IUCN Red List of Threatened Species. Available from: http://www.iucnredlist.org. (04 Nov 2006).

Kendrick, A. J. \& G. A. Hyndes. 2005. Variations in the dietary compositions of morphologically diverse syngnathid fishes. Environmental Biology of Fishes, 72:415-427.

Lourie, S. A., A. C. J. Vincent \& H. Hall. 1999. Seahorses: An identification guide to the world's species and their conservation. London, Project Seahorse, $214 \mathrm{p}$.

Lourie, S. A., S. J. Foster, E. W. T. Cooper \& A. C. J. Vincent. 2004. A Guide to the Identification of Seahorses. Project Seahorse and TRAFFIC North America. Washington D.C., University of British Columbia and World Wildlife Fund, 114p.

Lyons, D. O. \& J. J. Dunne. 2005. Reproductive ecology and operational sex ratio of Worm Pipefish (Nerophis lumbriciformis) in Irish waters. Proceedings of Royale Irish Academy, 105B(1):9-14

Martin-Smith, K. M. \& A. C. J. Vincent. 2005. Seahorse declines in the Derwest estuary, Tasmania, in the absence of fishing pressure. Biological Conservation, 123:533-545.

Martin-Smith, K. M. \& A. C. J. Vincent. 2006. Exploitation and trade in Australian seahorses, pipehorses, sea dragons and pipefishes (Family Syngnathidae). Oryx, 40:141-151.

Masonjones, H. D. 1997. Relative parental investment of male and female dwarf seahorses, Hippocampus zosterae. American Zoologist, 37:114A.

Masonjones, H. D. \& S. M. Lewis. 1996. Courtship behavior in the dwarf seahorse, Hippocampus zosterae. Copeia, 1996:634-640.

Masonjones, H. D. \& S. M. Lewis. 2000. Differences in potential reproductive rates of male and female seahorses related to courtship roles. Animal Behaviour, 59:11-20.

Moreau, M. A. \& A. C. J. Vincent. 2004. Social structure and space use in a wild population of the Australian short-headed seahorse Hippocampus breviceps Peters, 1869. Marine and Freshwater Research, 55:231-239.

Nijhoff, M. 1993. Life cycle of the seahorse Hippocampus reidi on the Bonaire coral reef. Dissertation, Unpublished Dissertation, Rijksuniversiteit Groningen, 50p.

Perante, N. C., A. C. J. Vincent \& M. G. Pajaro. 1998. Demographics of the seahorse Hippocampus comes in the central Philippines. Pp: 439-448. In: Morton, B. (Ed). Proceedings of the 3rd International Conference on the Marine Biology of the South China Sea. Hong Kong, Hong Kong University Press, 578p.

Perante, N. C., M. G. Pajaro, J. J. Meeuwig \& A. C. J. Vincent. 2002. Biology of a seahorse species Hippocampus comes in the central Philippines. Journal of Fish Biology, 60:821-837.

Roelke, D. L. \& S. M. Sogard. 1993. Gender-Based Differences in Habitat Selection and Activity Level in the Northern Pipefish (Syngnathus fuscus). Copeia, 1993(2):528-532

Rosa, I. L. 2005. National Report - Brazil. Pp. 46-53. In: Bruckner, A. W., J. D. Fields \& N. Daves (Eds.). The Proceedings of the International Workshop on CITES Implementation for Seahorse Conservation and Trade. Silver Spring, NOAA Techinical Memorandum NMFS-OPR-35, 178p.

Rosa, I. L., T. L. Dias \& J. K. Baum. 2002. Threatened fishes of the world: Hippocampus reidi Ginsburg, 1933 (Syngnathidae). Environmental Biology of Fishes, 64(4):378.

Rosa, I. L., R. R. N. Alves, K. M. Bonifácio, J. S. Mourão, F. M. Osório, T. P. R. Oliveira \& M. C. Nottingham. 2005. Fishers' knowledge and seahorse conservation in Brazil. Journal of Ethnobiology and Ethnomedicine, 1: 12.

Strawn, K. 1958. Life history of the pigmy seahorse, Hippocampus zosterae Jordan and Gilbert, at Cedar Key, Florida. Copeia, 1958:16-22.

Teixeira, R. L. \& J. A. Musick. 2001. Reproduction and food habits of the lined seahorse, Hippocampus erectus (Teleostei: Syngnathidae) of Chesapeake Bay, Virginia. Revista Brasileira de Biologia, 61:79-90.

Vari, R. P. 1982. The seahorses (Subfamily Hippocampinae). Pp. 173-189. In: Böhlke, J. M., D. M. Cohen, B. B. Collette, W. N. Eschmeyer, R. H. Gibbs-Jr, T. W. Pietsch, W. J. Richards, C. L. Smith \& K. S. Thomson (Eds.). Fishes of the Western North Atlantic, Part 8. Order Gasterosteiformes, Suborder Syngnathoidea. Syngnathidae (Doryrhamphinae, Syngnathinae, Hippocampinae). New Haven, Sears Foundation for Marine Research, 198p.

Vincent, A. C. J. 1990. Reproductive Ecology of Seahorses. Unplubished PhD Thesis, University of Cambridge, Cambridge, $101 \mathrm{p}$.

Vincent, A. C. J. 1994a. Seahorses exhibit conventional sex roles in mating competition, despite male pregnancy. Behaviour, 128:135-151.

Vincent, A. C. J. 1994b. Operational sex ratios in seahorses. Behaviour, 128:153-167.

Vincent, A. C. J. 1995. A role for daily greetings in maintaining seahorse pair bonds. Animal Behaviour, 49:258-260.

Vincent, A. C. J., K. L. Evans \& A. D. Marsden. 2005. Home range behaviour of the monogamous Australian seahorse, Hippocampus whitei. Environmental Biology of Fishes, 72(1):1-12.

Vincent, A. C. J. \& B. G. Giles. 2003. Correlates of reproductive success in a wild population of Hippocampus whitei. Journal of Fish Biololgy, 63:344-355. 
Vincent, A. C. J. \& L. M. Sadler. 1995. Faithful pair bonds in wild seahorses, Hippocampus whitei. Animal Behaviour, 50:15571569.

Watanabe, S. \& Y. Watanabe. 2001. Brooding season, sex ratio, and brood pouch development in the seaweed pipefish, Syngnathus schlegeli, in Otsuchi Bay, Japan. Ichthyological Research, 48(2):155-160.

Woods, C. M. C. 2000. Preliminary observations on breeding and rearing the seahorse Hippocampus abdominalis (Teleostei: Syngnathidae) in captivity. New Zealand Journal of Marine and Freshwater Research, 34:475-485.

Zar, J. H. 1999. Biostatistical Analysis. New Jersey, Prentice Hall, 929 p.

Submitted February 2007

Accepted August 2007 